\title{
The effect of dietary supplements of $\omega 3$ polyunsaturated fatty acids on the fatty acid composition of platelets and plasma choline phosphoglycerides
}

\author{
BY T. A. B. SANDERS AND KATHERINE M. YOUNGER \\ Department of Nutrition, Queen Elizabeth College, Campden Hill Road, London W8 $7 A H$
}

(Received 7 October 1980 - Accepted 25 November 1980)

1. The effects of dietary supplements of $\omega 3$ polyunsaturated fatty acids on the fatty acid composition of platelets and plasma choline phosphoglycerides were studied in vegans and in omnivores.

2. A supplement of $18: 3 \omega 3$ led to an increase in $20: 5 \omega 3$ but was less effective than one of $20: 5 \omega 3+22: 6 \omega 3$.

Although it is not known if linolenic acid $(18: 3 \omega 3)$ is essential its derivatives are important (Tinoco et al. 1979). Eicosapentaenoic acid $(20: 5 \omega 3)$ is the precursor of the triene prostaglandins (Gryglewski et al. 1979) and when incorporated into platelet lipids may influence bleeding time (Sanders et al. 1980). Docosahexaenoic acid (22:6w3) is a major component of human brain and retinal lipids and is found in its highest concentrations in the phosphoglycerides of synaptic membranes and rod outer segments implying that it has a role in neural transmission.

Radiotracer experiments in vitro suggest that man can convert $18: 3 \omega 3$ to $20: 5 \omega 3$ and 22:6w3 (De Gomez Dumm \& Brenner, 1975; Aerberhard et al. 1978) but this capacity may be limited (Dyerberg et al. 1980). Consequently dietary 20:5 important (Crawford \& Sinclair, 1972), the only significant sources being fish, fish oil and offal (Shepherd et al. 1978; Southgate \& Paul, 1978). Indeed, vegans whose diets are devoid of 20:5 $\omega 3$ and 22:6 33 have very much lower proportions of these fatty acids in their plasma choline phosphoglycerides than do omnivores (Sanders et al. 1977). It was suggested that the high value for the ratio, $18: 2 \omega 6: 18: 3 \omega 3$ in the vegan diets suppressed the transformation of $18: 3 \omega 3$ to $20: 5 \omega 3$ and $22: 6 \omega 3$ and instead favoured the conversion of $18: 2 \omega 6$ to $20: 4 \omega 6$ and 22:4 $\omega 6$. This being so then a dietary supplement of $18: 3 \omega 3$ should reduce the value

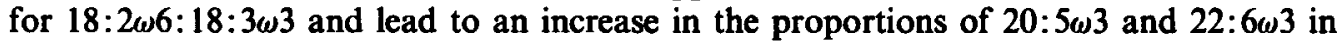
this lipid fraction. In order to test this hypothesis, vegan and omnivore subjects were given a supplement of linseed oil, rich in 18:3 33 for 2 weeks and changes in their blood lipids were monitored. So that a comparison could be made between dietary linolenate and its

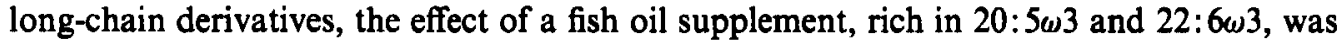
also studied in the omnivores.

\section{EXPERIMENT AL}

\section{Subjects, materials and methods}

Vegan subjects (aged 26-37 years) were contacted through the Vegan Society (47 Highlands Road, Leatherhead, Surrey) and omnivores (aged 23-47 years) were recruited from the staff and students of the Nutrition Department, Queen Elizabeth College. All the subjects considered themselves to be healthy. Consent was obtained from individuals and from the College Ethical Committee for all procedures.

In the first experiment, four vegan (three male, one female) and five omnivore subjects (three male, two female) were instructed to take $5 \mathrm{ml}$ refined and deoderized linseed oil four times daily with meals for 2 weeks. In the second experiment, two male and two female omnivore subjects, who had 7 weeks previously taken the linseed oil supplement, took a 
Table 1. Fatty acid composition (wt \%) of the two oils fed to the subjects

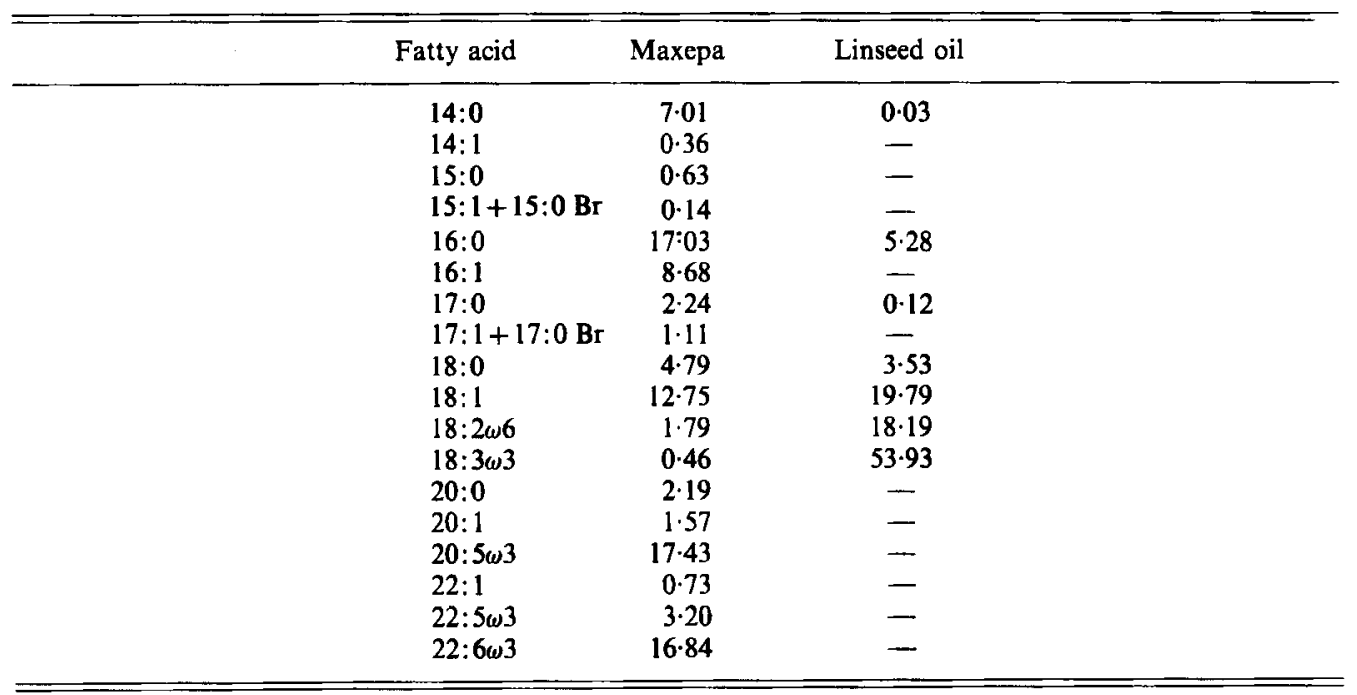

fish oil supplement (Maxepa) for 2 weeks ( $5 \mathrm{ml}$ four times daily with meals). The oils used were provided by British Cod-liver Oils, Hull and were analysed for fatty acids by gas-liquid chromatography before use (Table 1). Fresh bottles of oil were issued each week and oil consumption was assessed by weighing the bottles. All subjects completed a 7-d-weighed dietary intake record. Nutrient intakes were calculated by computer using the tables of Southgate \& Paul (1978). For foods not included in these tables additional values were obtained from other published sources (Platt, 1962; Watt \& Merrill, 1963; Miller \& Mumford, 1972; US Department of Health \& Welfare \& FAO, 1972; Shepherd et al. 1978) or from information provided by the manufacturers of proprietary products.

Venous blood samples were drawn from subjects after an overnight fast with minimum venostasis before and after taking the supplements. The blood samples were anticoagulated with EDTA, chilled to $4^{\circ}$ and processed immediately. Platelet and plasma choline phosphoglycerides (Sanders et al. 1978) were prepared and transmethylated with sodium methoxide after elution from the thin-layer chromatography absorbent (Christie, 1973). The resulting methyl esters were anlysed by gas-liquid chromatography on a Pye model 204 chromatograph equipped with flame ionization detectors: a $1.8 \mathrm{~m} \times 4 \mathrm{~mm}$ internal diameter glass column packed with $100 \mathrm{~g}$ Silar $10 \mathrm{C} / \mathrm{kg}$ Gaschrom Q (100-120 mesh) was used; oven temperature $200^{\circ}$, injection temperature $210^{\circ}$ and detector temperature $260^{\circ}$; nitrogen carrier gas flow-rate $40 \mathrm{ml} / \mathrm{min}$. Chromatographic peaks were integrated using a DP 88 integrator (W. G. Pye, Cambridge). Methyl esters were identified by comparison with mixtures of known composition. Analyses were confirmed on a less polar column packed with $100 \mathrm{~g}$ Silar $5 \mathrm{C} / \mathrm{kg}$ Chromosorb W HP (80-100 mesh) operated at $210^{\circ}$. Statistical analyses of the results were performed using a one-tailed paired sample $t$ test, with each subject acting as his or her own control. A two-sample two-tailed $t$ test was used for between group comparisons.

RESULTS AND DISCUSSION

The vegans had followed their diet for an average of 10 years (range 5-14). Their diets comprised unrefined cereals, nuts, fruit, pulses and vegetables and were supplemented with 
vitamin $B_{12}$. Such diets do not result in detectable deficiency (Sanders, 1978). Their mean energy intake was $10 \mathrm{MJ}$ (range 6-14 MJ) compared with $8 \mathrm{MJ}$ (range 6-10 MJ) in the omnivores; all the diets provided at least one-tenth of the dietary energy as protein. Although their average fat intake was similar to that of the omnivores $(84 \pm 5 v .87 \pm 5 \mathrm{~g} / \mathrm{d})$, its composition was very different: their intake of saturated fatty acids was much lower $(16 \pm 1 v .38 \pm 3 \mathrm{~g} / \mathrm{d}$ in the omnivores) and that of linoleic acid $(18: 2 \omega 6)$ was almost four times greater $(28 \pm 2 v .7 \pm 1 \mathrm{~g} / \mathrm{d})$; their average intake of linolenic acid (18:3w3) was similar $(2 \cdot 0 \pm 0.6$ v. $1 \cdot 3 \pm 0.2 \mathrm{~g} / \mathrm{d})$. Therefore, the ratio, $18: 2 \omega 6: 18: 3 \omega 3$ was much greater in the vegans than in the omnivores $(16 \pm 3 \cdot 5$ v. $6 \pm 1 \cdot 0)$. This high value would be expected to suppress the conversion of $18: 3 \omega 3$ to its long-chain derivatives. Indeed, the differences in the fatty acid composition of the plasma choline phosphoglycerides between vegans and omnivores were similar to those previously reported (Sanders et al. 1978): the proportion of 20:5 $\omega 3$ and especially $22: 6 \omega 3$ were much lower (Table 2) and those of $20: 4 \omega 6(13.9 \pm 0.31$ v. $11.3 \pm 0.65 \mathrm{wt} \% ; P<0.05)$ and $18: 2 \omega 6(29.7 \pm 1.5 v .24 .5 \pm 1.39 \mathrm{wt} \% ; P<0.05)$ were greater. Comparable differences were noted in the platelet phosphoglycerides.

Table 2. Proportions (wt \%) of eicosapentaenoic (20:5w3) and docosahexaenoic (22:6w3) acids in plasma choline phosphoglycerides (CPG) and platelet phosphoglycerides (PG) of vegans and omnivores before and after 2 weeks of taking an oil supplement

(Results are expressed as mean values with their standard errors)

\begin{tabular}{|c|c|c|c|c|c|c|c|c|c|c|}
\hline \multirow[b]{3}{*}{ Group } & \multirow{3}{*}{$\begin{array}{c}\text { No. } \\
\text { of } \\
\text { subjects }\end{array}$} & \multirow[b]{3}{*}{ Supplement } & \multicolumn{4}{|c|}{$20: 5 \omega 3$} & \multicolumn{4}{|c|}{$22: 6 \omega 3$} \\
\hline & & & \multicolumn{2}{|c|}{ Before } & \multicolumn{2}{|c|}{ After } & \multicolumn{2}{|c|}{ Before } & \multicolumn{2}{|c|}{ After } \\
\hline & & & Mean & $\mathbf{S E}$ & Mean & SE & Mean & SE & Mean & SE \\
\hline \multicolumn{11}{|l|}{ Plasma CPG } \\
\hline Omnivores & 5 & Linseed oil & $1 \cdot 3$ & 0.37 & $2 \cdot 7 *$ & 0.50 & $4 \cdot 3$ & 0.33 & 3.7 & 1.03 \\
\hline Vegans & 4 & Linseed oil & $0.3 \dagger$ & 0.06 & $1 \cdot 0^{*} \dagger$ & 0.24 & $1 \cdot 3+$ & 0.30 & $1.3+$ & 0.46 \\
\hline Omnivores & 4 & Maxepa $\ddagger$ & 1.5 & 0.27 & $6 \cdot 7^{*}+$ & 0.96 & 3.9 & 0.45 & $4 \cdot 5^{*}$ & 0.34 \\
\hline \multicolumn{11}{|l|}{ Platelet PG } \\
\hline Omnivores & 5 & Linseed oil & 0.6 & 0.10 & $1 \cdot 2^{*}$ & $0 \cdot 18$ & 3.0 & 0.33 & 2.9 & $0-09$ \\
\hline Vegans & 4 & Linseed oil & $0.3 \dagger$ & 0.04 & 0.37 & 0.04 & $0.9 \dagger$ & $0 \cdot 17$ & $1 \cdot 2 \dagger$ & 0.26 \\
\hline Omnivores & 3 & Maxepał & $1 \cdot 2$ & $0 \cdot 17$ & $4 \cdot 1 *+$ & 0.10 & $2 \cdot 7$ & 0.26 & $3.9 * \dagger$ & 0.27 \\
\hline
\end{tabular}

Statistical significance of difference between mean values; $* P<0.05$ compared with before the supplement; $\dagger P<0.05$ compared with the corresponding values obtained for the omnivores who took the linseed oil supplement.

$\ddagger$ British Cod-liver Oils, Hull.

The linseed oil supplement, which provided an average of $6.5 \mathrm{~g} 18: 3 \omega 3 / \mathrm{d}$, reduced the

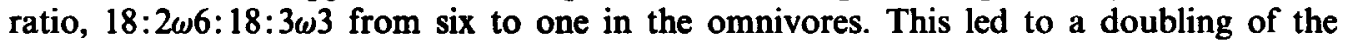
proportion of $20: 5 \omega 3$ in both plasma choline phosphoglycerides and in the platelets. The same supplement reduced the value for the ratio from sixteen to three in the vegans and led to a threefold increase in the proportion of 20:5 3 in the plasma choline phosphoglycerides but not in the platelets. These observations provide evidence that man can convert 18:3 $\omega 3$ to $20: 5 \omega 3$. The higher proportion of linoleic acid in the adipose tissue of vegans compared with omnivores (Sanders et al. 1978) would tend to buffer any short-term attempt to reduce the ratio $18: 2 \omega 6: 18: 3 \omega 3$ in the body. Consequently a more marked increase in the proportion of 20:5w3 might have occurred in the vegans had the supplement been continued for longer. 
The failure of the linseed oil supplement to increase the proportion of 22:6 33 in these lipid fractions in both vegans and omnivores is consistent with the hypothesis that the rate of $\Delta 4$ desaturation is slow in man (Sanders \& Naismith, 1979).

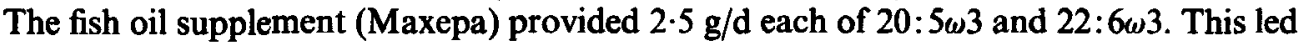
to a fourfold increase in the proportion of $20: 5 \omega 3$ and a less marked increase in 22:6 3 in both the plasma choline phosphoglycerides and the platelets in the omnivores; these changes occurred mainly at the expense of $18: 2 \omega 6$ in the plasma choline phosphoglycerides

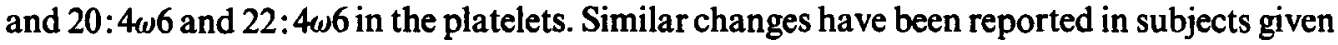
a cod-liver oil supplement (Sanders et al. 1980). The relatively small amounts of 20:5w3 and 22:6 $\omega 3$ provided by the Maxepa supplement had a far greater effect than the larger amount of 18:3 $\omega 3$ provided by the linseed oil. Thus the effect of administering different

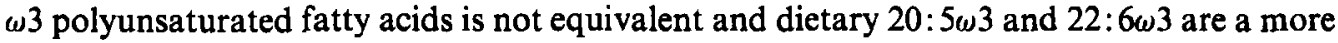
effective means of increasing the proportions of these acids in tissue lipids than a reduction in the ratio $18: 2 \omega 6: 18: 3 \omega 3$ in the diet. We were unable to make a trustworthy estimate of the $20: 5 \omega 3$ and $22: 6 \omega 3$ in the diets of the omnivore subjects owing to the lack of reliable food composition information. However, our results imply that the major source of 22:6 33 in membrane phospholipids is obtained preformed rather than indirectly from dietary linolenic acid. This would explain the higher proportion of 22:6w3 in the lipids of the omnivores compared with the vegans. Further research is necessary to determine the optimum levels of $\mathrm{C}_{20-22} \omega 3$ polyunsaturated fatty acids in tissues.

The authors are grateful to British Cod-liver Oils.

\section{REFERENCES}

Aerberhard, E. E., Corbo, L. \& Menkes, J. H. (1978). Lipids 13, 758.

Christie, W. W. (1973). Lipid Analysis. Oxford: Pergamon.

Crawford, M. A. \& Sinclair, A. J. (1972). In Lipids, Malnutrition and the Developing Brain, p. 267 [K. Elliot and J. Knight, editors]. Amsterdam: Elsevier.

De Gomez Dumm, I. N. T. \& Brenner, R. R. (1975). Lipids 10, 315.

Dyerberg, J., Bang, H. O. \& Aagard, O. (1980). Lancet i, 199.

Gryglewski, R. J., Salmon, J. A., Ubatuba, F. B., Weatherley, B. C., Moncada, S. \& Vane, J. R. (1979). Prostaglandins 18, 453.

Miller, D. S. \& Mumford, P. (1972). Pl. Fd hum. Nutr. 2, 201.

Platt, B. S. (1962). Med. Res. Counc. Spec. Rep. Ser. no. 302.

Sanders, T. A. B. (1978). Pl. Fds Man 2, 181.

Sanders, T. A. B., Ellis, F. R. \& Dickerson, J. W. T. (1977). Lancet i, 751.

Sanders, T. A. B., Ellis, F. R. \& Dickerson, J. W. T. (1978). Am. J. clin. Nutr. 31, 805.

Sanders, T. A. B., Haines, A. P. \& Vickers, M. (1980). Lancet i, 1189.

Sanders, T. A. B. \& Naismith, D. J. (1979). Br. J. Nutr. 41, 619.

Shepherd, A. J., Iverson, J. \& Weihrauch, J. L. (1978). In Handbook of Lipid Research, vol. 1, p. 341 [A. Kuksis, editor]. London: Plenum Press.

Southgate, D. A. T. \& Paul, A. A. (1978). McCance \& Widdowson's The Composition of Foods. London HM Stationery Office.

Tinoco, J., Babcock, R., Hincenbergs, I., Medwadowski, B. \& Miljanich, P. (1979). Lipids 14, 166.

US Department of Health \& Welfare \& FAO. (1972). Food Composition Tables for Use in S.E. Asia. Rome: FAO.

Watt, B. K. \& Merrill, A. G. (1963). Composition of foods. Handbook no. 8. Washington: US Department of Agriculture. 